REVIEW

\title{
GONADOTROPIN-RELEASING HORMONE AGONISTS IN ENDOMETRIOSIS TREATMENT AND IN VITRO FERTILIZATION
}

\author{
Sofia Lider-Burciulescu ${ }^{1}$, Romina-Marina Sima ${ }^{1,2}$, Liana Pleş ${ }^{1,2}$ \\ 'The University of Medicine and Pharmacy "Carol Davila", Bucharest, Romania \\ “"St. John” Hospital, "Bucur Maternity”, Bucharest \\ Corresponding author: Romina Sima \\ Phone no. 0040741071243 \\ E-mail: romina.sima@yahoo.es
}

\begin{abstract}
Endometriosis is a chronic disease of unknown etiology defined as the presence of ectopic endometrial and it affects approximately $10 \%$ of women in reproductive age. Sequelae of endometriosis are represented by infertility and chronic pelvic pain. GnRHa (gonadotropin releasing hormone) represent one of one medical approaches that were tried for endometriosis treatment.Choosing between agonists and antagonists means to compare risks and benefits. The antagonists of GnRH are less used because of their many side effects. GnRH agonists have many advantages such as: lower cost, less side effects and shorter treatment. The GnRH therapy is not only useful in the treatment of endometriosis but also in IVF. Nowadays, researches shows that the laparoscopic ablation is as efficient as the GnRH agonists therapy, but also a postoperative therapy with GnRH is indicated. Several protocols are actually available for IVF and Embrio Transfer. Also recent researches proved that a postoperative therapy with GnRH agonists in patients with endometriosis associated infertility raised the pregnancy rate and reduced pelvic pain.GnRH agonists can be considered as a first line therapy when severe pain is involved or second-line treatment in endometriosis in case of failure of therapy with oral contraceptives or progestins. GnRH is more efficient in the protocol of FIV than in the treatment of endometriosis.
\end{abstract}

Keywords: endometriosis, gonadotropin, in vitro fertilization

\section{Introduction}

The human body is a complex of interactions between mechanism and substances. Gonadotropin releasing hormone $(\mathrm{GnRH})$ or luteinizing hormone releasing hormone, or Luberin is one of the hormones secreted in the hypothalamus. In fact, GnRH is a neurohormone which is secreted by the neuronal cells situated in the preoptic area. In fetus, the GnRH neurons are found in nose and later they migrate into the brain.

GnRH was discovered in 1971 by Schally and Guillemin. After them, there were identified many types of isohormones in human beings and also at other vertebrates. In human, there were isolated three types of GnRh. Among this, GnRH type 1 is the best known and it is composed of 10 aminoacids. Aminoacids substitutions have revealed the significance of specific regions for stability, receptor binding and activation of the pituitary gonadotropins. Changes, mainly in position 6 and 10, give rise to analogues with increased potency, extending the half life from minutes to hours and raising the binding capacity more than 100 folds. The gene that initiated the production of $\mathrm{GnRH}$ is situated on the chromozome 8 [1]. 
In physiological conditions the action of $\mathrm{GnRH}$ is manifested after its releasing by the hypothalamus into the portal system and transported into the pituitary gland. Here, it stimulates the GnRH receptors of the gonadotropin cells. After that, the gonadotropin cells release the FSH (Foliculle Stimulating Hormone) and LH (Luteinizing Hormone).

The mechanism of action of $\mathrm{GnRH}$ is the same for all mediators with the same affinity for $\mathrm{G}$ protein-coupled receptors. The receptor has an extramembrane part, seven transmembrane parts and an intramembrane part witch is cuplated with $\mathrm{G}$ protein. Binding $\mathrm{GnRH}$, the receptor activates the $G$ protein and then the second messenger like IP3 (inositol phosphate) and DAG (diacyl glicerol) are activated. Their activation leads to the activation of PKC (Protein Kinase C). The outcome of this mechanism is the releasing of calcium from the RER (Rugos Endoplasmic Reticulum). That is how FSH and LH are produced. It is necessary to underline that the secretion of $\mathrm{GnRH}$ is pulsatory in women and liniary in men [2].

$\mathrm{GnRH}$ is very important in controling the women`s hormonal status, as multiple researches show on the matter of the use of synthetic GnRH for the treatment of dysfunction of the female gonads [3].

The first pilot study for utilization of GnRH agonists started in 1980 in research for the treatment of endometriosis. It is also demonstrated that the utilization of $\mathrm{GnRH}$ agonists is more efficient in the treatment of ovarian endometriosis than following a surgical method [4].

Active endometriosis is dependent on the estrogen stimulation. When GnRH agonists are used in the treatment of endometriosis, the levels of $\mathrm{FSH}$ and $\mathrm{LH}$ are increased in the first 2 weeks, but afterwards the phenomenon of down regulation appears and the response of hypophisis at $\mathrm{GnRH}$ decrease. The result is a decrease of the estrogens blood level at less than 50 microgrames after 14-21 days of treatment [5].

\section{GnRH and In Vitro Fertilisation (IVF)}

In 1994, Diedrich and all observed that the utilization of $\mathrm{GnRH}$ decreases the rate of abnormal cycles and it allows to schedule the moment of ovulation. Nowadays it is demonstrated that the utilization of $\mathrm{GnRH}$ agonist has more advantages than the utilization of gonadotropin, which increases the level of LH [6]. The contribution of GnRH agonists has as drawback the following side-effects: vasomotor symptoms and bone demineralization [7].

That is the reason for introduction of addback therapy in the treatment with GnRH of endometriosis. It consists of administration of estrogens in order to avoid the side effects produced by the drop of the estrogen levels.

Endometriosis is a condition not fully understood thus without an ethiological cure. Many treatments were proposed in the past decades. Patients with chronic pelvic pain need many courses of medical therapy or even surgical therapy, or both. In many cases, the empirical treatment only with GNRH is not effective [8].

\section{Surgical approaches or GnRH agonists}

Surgical approaches should be the first intention for stage III or stage IV endometriosis, when pelvic pain is invalidant or if the medical therapy failed [9]. The most used surgical method is the laparoscopic ablation of the endometriotic sites. At the beginning of the $21 \mathrm{st}$ century, research showed that the simple surgical therapy is more efficient than a postsurgical therapy with GnRH agonists. Nowadays, research shows that the laparoscopic ablation is as efficient as the GnRH agonists therapy, but also a postoperative therapy with GnRH is indicated [10].

The GnRH therapy is not only useful in the treatment of endometriosis but also in IVF. Several protocols are actually available for IVF and Embrio Transfer. The studies indicate that a combination between exogenous gonadotropin and GNRH agonists which is able to suppress pituitary $\mathrm{FSH}$ and $\mathrm{LH}$ secretion is associated with an increased pregnancy rate as compared to the use of gonadotropins without GnRH agonists [11]. 


\section{The role of GnRH in IVF}

GnRH agonists are usually part of IVF treatment. In $1980 \mathrm{GnRH}$ was used for prevention of $\mathrm{LH}$ surge during the ovarian stimulation. By the end of the 1989, the long down regulation with GnRH was used for IVF practice and the protocol for ART was established [12].

Over decades, there were many studies about the utilization of GnRH agonists in IVF protocol. In the long protocol, it starts in the luteal phase of the run-in cycle and continues until the ovulatory trigger. Its agonistic mechanism produces an initial flare of gonadotrophin release before the receptors are down regulated. Stimulation starts about 2 weeks later, when the endometrium from the run-in-cycle has been well shed. The protocol is flexible at this stage as a few more days of down regulation do not affect the subsequent stimulation or cycle outcome to any significant degree. GnRH agonists induce the supression of endogenous releasing of gonadotropin during the early follicular phase allowing the early antral follicles to grow as a response to exogenous gonadotropins. That leads to an wider FSH window, and increased number of recruited mature follicles and a higher number of retrieved ovocytes. Some women will experience hypoestrogenic side effects of varying severity during the time [13].

Choosing between agonists and antagonists means to compare risks and benefits, The antagonists of $\mathrm{GnRH}$ are less used because of their many side effects. GnRH agonists have many advantages such as: lower cost, fewer side effects and shorter treatment. But the antagonists cause an immediate suppression of gonadotropin secretion, without initial stimulatory effect [14].

Many studies compared the efficiency between simple surgical approaches and the GnRH agonists. Nowadays some studies suggest that a postoperative therapy with $\mathrm{GnRH}$ agonists in patients with endometriosis associated infertility raised the pregnancy rate and reduced the pain [15].

A recent Cochrane study suggests that women with endometriosis undergoing assisted reproduction after three months of treatment with $\mathrm{GnRH}$ agonist improved their pregnancy rates. In the same article it is mentioned that the excisional surgery improved spontaneous pregnancy rates in the following 9 to 12 months after surgery compared to the ablative surgery [16].

\section{Endometriosis and GnRh agonists}

\section{Signs and symptoms of endometriosis}

Endometriosis is defined as the presence of ectopic endometrial tissue with specific symptoms and inflammatory reaction. (Figure 1) It is a chronic disease of unknown etiology that affects approximately $10 \%$ of women in the reproductive age [17]. The main sequelae of endometriosis are represented by infertility and chronic pelvic pain. Up to $40 \%$ of infertile women and one-third of women who undergo laparoscopy for chronic pelvic pain have endometriosis [18]. The diagnosis of endometriosis is based on many investigations, and is based on the symptoms and signs. But the gold standard for the diagnosis of endometriosis is laparoscopy. The pathologic exam is mandatory but sometimes it cannot be obtained. In the presence of the specific lesions in laparoscopy the diagnosis can be established even if the pathologic examination is unclear. The correct laparoscopic diagnosis depends on the ability of the surgeon.

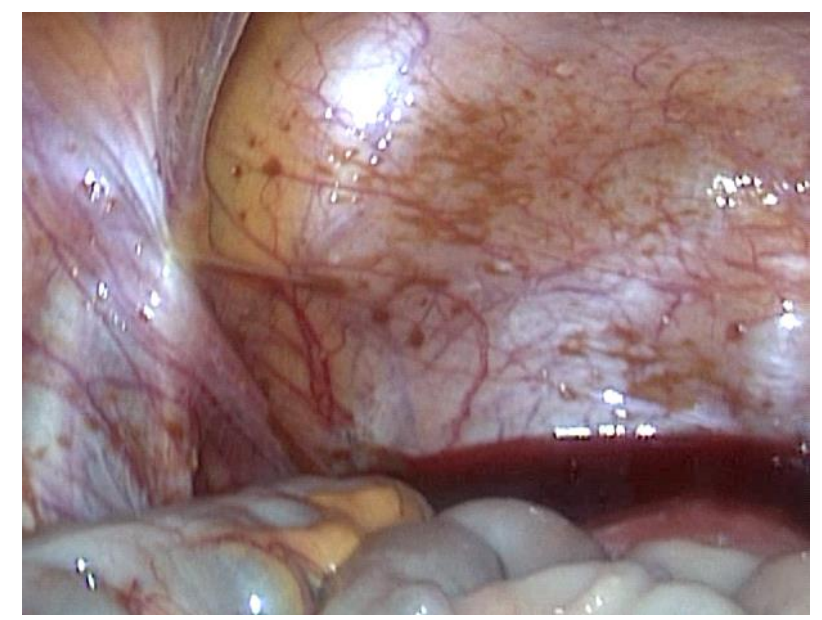

Figure 1 - Laparoscopy: Peritoneal endometriosis

Pelvic pain: cyclic pelvic pain, dyspareunia and dysmenorrhea are the most specific symptoms of endometriosis. Others symptoms such as fatigue and infertility were reported. But the most common symptom is dysmenorrhea (62\%) [19]. Also, the symptoms are dependent 
on the location of the disease. Deep endometriosis in the posterior pelvis is associated with increased severity of dyschezia. Intestinal symptoms are reported but these are not specific. Adolescent girls with endometriosis report a high rate of symptoms: uterine cramping, cyclic pain, constipation or diarrhea [20].

The diagnosis of endometriosis may include the following gynecological symptoms: dysmenorrhea, cyclical pelvic pain, deep dyspareunia, infertility.

And non-gynecological symptoms: dyscezia, dysuria, hematuria, rectal bleeding or shoulder pain [17]. (Figure 2)

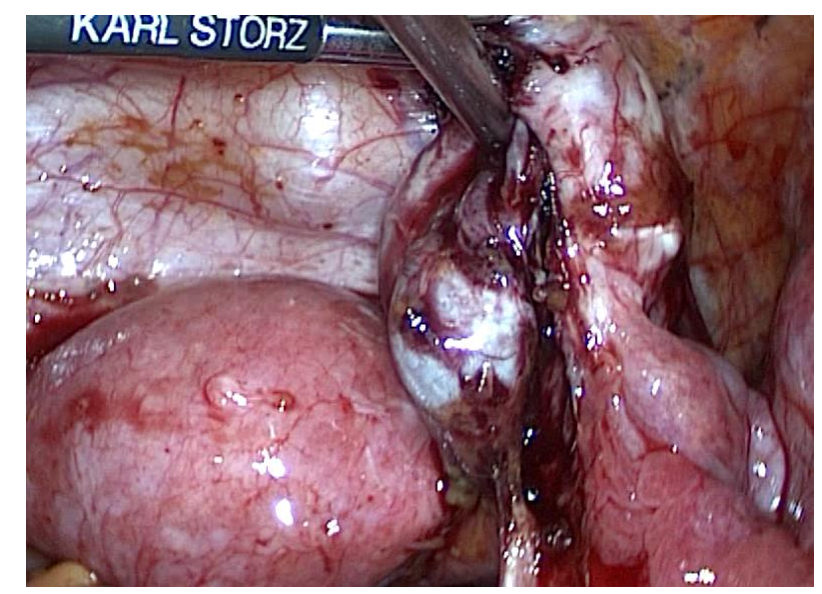

Figure 2 - Laparoscopy: Ovarian endometriosis

\section{Clinical examination in endometriosis}

Diagnosis of endometriosis can be suspected by vaginal examination. But this exam may be inappropriate in teenagers and women without previous sexual intercourse. In those situation a rectal examination is preferable [17].

The diagnosis may be sustained by the presence of induration and nodules of the posterior vaginal fornix and of the rectovaginal wall, or in women with adnexal masses. Clinicians must consider the diagnosis of endometriosis even if the physical signs are not detected, but the symptoms are reported.

Paraclinical examinations are also used for endometriosis diagnosis. TVS (transvaginal sonography) is an useful method that can identify ovarian endometriosis. 3D sonography is not very useful. In many units diagnosis of endometriosis rely on specific signs on magnetic resonance imaging which can also distinguish between ovarian endometriosis and other ovarian tumors.

Biomarkers such as CA-125 in urine and serum can be used but are not specific for the diagnosis of endometriosis [21].

\section{Hormonal therapies in endometriosis associated pain}

Endometriosis is a estrogen-dependent condition. In time, many studies tried to demonstrate the efficacity of hormonal suppression with hormonal contraceptives, GNRH agonists and aromatase inhibitors [22]. (Figure 3)

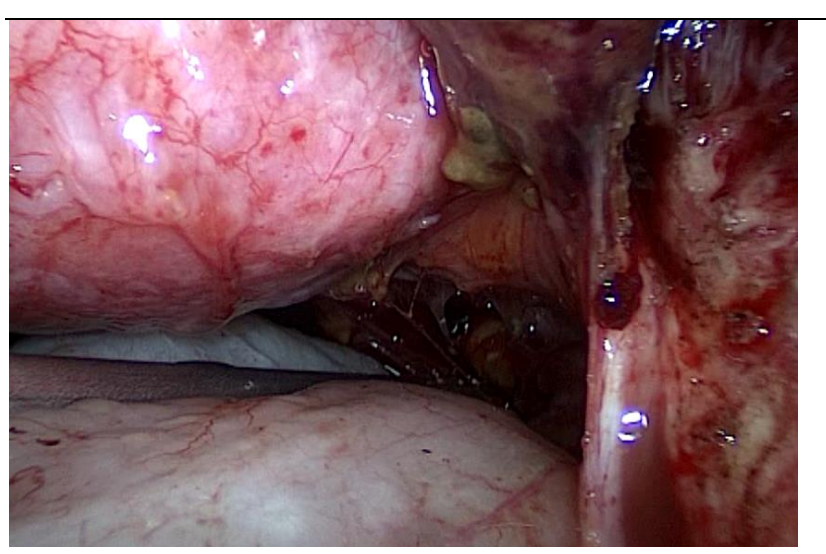

Figure 3 - Laparoscopy: Deep infiltrating endometriosis

\section{GnRh agonists and endometriosis}

The efficacity of GnRHa was demonstrated in studies which compared their effective role with placebo and analgesics. GnRHa are more useful than placebo but less useful than oral danazol and levonorgestrel- releasing intrauterine system. There is no difference between the intramuscular, subcutaneous or intranasal administration of GnRHa. A difference is demostrated for the short protocol (3 months) with GNRHa: dyspareunia has decreased in the shorter protocol [23].

\section{Preoperative treatment of endometriosis}

Despite the fact that most clinicians use preoperative GnRH agonists their utility was not demonstrated by studies [24].

Some clinicians consider that a preoperative treatment with GnRHa reduce the inflammation and the rate of bleeding during the surgery [25]. 


\section{Postoperative hormonal therapy of endometriosis.}

Hormonal postoperative therapy is prescribed in two situations:

- Adjuvant therapy within 6 months after surgery;

- Secondary prevention of the recurrence of pain symptoms.

The most used postoperative therapy is levonorgestrel- releasing intrauterine system or combined hormonal contraceptives [26].

\section{Conclusion}

GnRH analogues (GnRH-a) suppress estrogen ovarian production through a downregulation of $\mathrm{GnRH}$ receptors at the pituitary level. The effect is reversible after stopping the GnRH-a administration. They can be considered as a first line therapy when severe pain is involved or second-line treatment in endometriosis in case of failure of therapy with oral contraceptives or progestins. GnRH analogues provide a reduction of symptoms and their administration after surgical treatment can extend the pain-free interval. GnRH is more efficient in the protocol of FIV than in the treatment of endometriosis.

\section{References}

[1]André C.D. van Loenen, MD, Judith A.F. Huirne, MD, Roel Schats, MD, PhD, Peter G.A. Hompes, $\mathrm{MD}, \mathrm{PhD}$, Cornelis B. Lambalk, MD "GnRH Agonists, Antagonists, and Assisted Conception"Seminars in Reproductive Medicine-2002;20(4)

[2]Ortmann O1, Weiss JM, Diedrich K Gonadotrophin-releasing hormone $(\mathrm{GnRH})$ and GnRH agonists: mechanisms of action..-Reprod Biomed Online. 2002;5 Suppl 1:1-7.

[3]Robert D. Utiger, M.D-Gonadotropin-releasing hormone (GnRH) -Encyclopaedia Britannica-2009

[4]David L. Olive,M.D. -Gonadotropin-Releasing Hormone Agonists for Endometriosis, New England Journal of Medicine (NEJM)-2008; 359:1136-1142 September 11,2008

[5]Kamran S. Moghissi,MD-A Clinician's Guide to the Use of Gonadotropin-Releasing Hormone Analogues in Women-Medscape General Medicine Obs/Gyn and Women's Health-2000;2

[6]Dr. Muresan Maria Cezara-Analogii-GnRH in reproducerea umana asistataTehnici actuale in reproducerea umana asistata - ICSI si crioconservarea - Scribd 2008

[7]Surrey ES.Gonadotropin-releasing hormone agonist and add-back therapy: what do the data show?--Curr Opin Obstet Gynecol 2010 Aug

[8]Linda C. Giudice, M.D., Ph.D. -Endometriosis$\mathrm{N}$ Engl J Med 2010; 362:2389-2398June 24, 2010DOI: 10.1056/NEJMcp1000274

[9]Busacca M1, Somigliana E, Bianchi S, De Marinis S, Calia C, Candiani M, Vignali M. Postoperative $\mathrm{GnRH}$ analogue treatment after conservative surgery for symptomatic endometriosis stage III-IV: a randomized controlled trial.-Hum Reprod. 2001 Nov;16(11):2399-402.

[10]Byung Chul Jee,M.D.a,Joong Yeup Lee,M.D.b,Chang Suk Suh,M.D.a,cSeok Hyun Kim,M.D.c,Young Min Choi,M.D.c,Shin Yong Moon,M.D-,Impact of GnRH agonist treatment on recurrence of ovarian endometriomas after conservative laparoscopic surgery-Fertility and Sterility Volume 91, Issue 1, Pages 40-45, January 2009

[11]Bart C.J.M. Fauser, Geeta Nargund, Anders Nyboe Andersen, Robert Norman, Basil Tarlatzis, Jacky Boivin, William Ledger -Mild Ovarian Stimulation for IVF: 10 Years Later-Hum Reprod.2010;25(11):2678-2684.

[12]Donnez J.Chantraine F, Nisolle M.- The efficacy of medical and surgical treatment of endometriosisassociated infertility: arguments in favour of a medico-surgical aproach - Human Reproduction Update, Vol.8, No.1 pp. 89 $\pm 94,2002$

[13]Raffaella Depalo, K Jayakrishan, Gabriella Garruti, Ilaria Totaro, Mariantonietta Panzarino, Francesco Giorgino and Luigi E.-GnRH agonist versus GnRH antagonist in invitro fertilization and embryo transfer (IVF/ET)- Reproductive Biology and Endocrinology 2012, 10:26

[14]A. Pellicer, E. Labarta, E. Bosch - Agonist or antagonist. What is Preferable??-Instituto Universitario IVI (Instituto Valenciano de Infertilidad), Valencia, Spain 16

[15]Dagmar Rickes, M.D.Ingrid Nickel, M.D.Siegfried Kropf, Ph.D.Jürgen Kleinstein, M.DIncreased pregnancy rates after ultralong postoperative therapy with gonadotropin-releasing hormone analogs in patients with endometriosis.Fertility and Sterility Volume 78, Issue 4, Pages757762, October 2002-Presented at the Eighth World Congress on Endometriosis, San Diego, California, February 24-27, 2002.

[16]Brown J1, Farquhar C. Endometriosis: an overview of Cochrane Reviews.Cochrane Database Syst Rev. 2014 Mar 10;3:CD009590. doi: 10.1002/14651858.CD009590.pub2.

[17] Giudice LC, Kao LC. Endometriosis. The Lancet. 2004;364(9447):1789-1799 
[18]Guo S, Wang Y. The prevalence of endometriosis in women with chronic pelvic pain. Gynecologic and Obstetric Investigation. 2006;62(3):121-130

[19]Dunselman GA, Vermeulen N, Becker C, Calhaz-Jorge C, D'Hooghe T, De Bie B, Heikinheimo O, Horne AW, Kiesel L, Nap A, Prentice A, Saridogan E, Soriano D, Nelen W.Guideline of the European Society of Human Reproduction and Embryology -ESHRE Endometriosis Guideline Development Group -Hum Reprod. 2014 Mar;29(3):400-12

[20]Gülden Halis, Dr. med. Sylvia Mechsner, Dr. med.and Andreas D. Ebert, Prof. Dr. med.The Diagnosis and Treatment of Deep Infiltrating Endometriosis-Dtsch Arztebl Int. Jun 2010; 107(25): 446-456.

[21]Robert Z. Spaczynski, Antoni J. Duleba.Diagnosis of Endometriosis- Semin Reprod Med 2003; 21(2): 193-208
[22]David L. Olive, M.D., and Elizabeth A. Pritts, M.D.-Treatment of Endometriosis-N Engl J Med 2001; 345:266-275

[23]Christopher Sutton,Kevin Jones-EndometriosisCambridge University Press -May 2004

[24]Muzii L, Marana R, Caruana P, Mancuso S-The impact of preoperative gonadotropin-releasing hormone agonist treatment on laparoscopic excision of ovarian endometriotic cysts.-Fertility and Sterility [1996, 65(6):1235-1237]

[25]Susan Furness,Christine Yap,Cindy

Farquhar,Ying $\mathrm{C}$ Cheong-Pre and post-operative medical therapy for endometriosis surgery-Cochrane Menstrual Disorders and Subfertility Group-19 JUL 2004

[26]Somigliana E, Vercellini P, Vigano P, Benaglia L, Busnelli A, Fedele L-.Postoperative medical therapy after surgical treatment of endometriosis: from adjuvant therapy to tertiary prevention.-J Minim Invasive Gynecol. 2014 May-Jun;21(3):32834 